2. Purpura DP. Dendritic spine « dysgenesis » and mental retardation 1 . Science 1974 ; 186 : 1126-8.

3. Komiyama NH, Watabe AM, Carlisle HJ, et al. SynGAP regulates ERK/MAPK signaling, synaptic plasticity, and learning in the complex with postsynaptic density 95 and NMDA receptor. J Neurosci 2002 ; 22 : 9721-32.

4. Rumbaugh G, Adams JP, Kim JH, Huganir RL. SynGAP regulates synaptic strength and mitogen-activated protein kinases in cultured neurons. Proc Natl Acad Sci USA 2006 ; 103 : 4344-51.
5. Carlisle HJ, Manzerra P, Marcora $\varepsilon$, Kennedy MB. SynGAP regulates steady-state and activitydependent phosphorylation of cofilin. J Neurosci $2008 ; 28: 13673-83$

6. Guo X, Hamilton PJ, Reish NJ, et al. Reduced expression of the NMDA receptor-interacting protein SynGAP causes behavioral abnormalities that model symptoms of schizophrenia. Neuropsychopharmacology 2009 ; $34: 1659-72$
7. Hamdan FF, Gauthier J, Spiegelman D, et al. Mutations in SYNGAPI in autosomal nonsyndromic mental retardation. $N$ Engl J Med 2009 ; $360: 599-605$.

8. Davidovic L, Tremblay S, Gravel M, et al. The fragile X syndrome: one protein missing and 1001 disoriented mRNAs. Med Sci (Paris) $2006 ; 22: 41-6$

9. Billuart P, Chelly J, Gilgenkrantz S. X-linked mental retardation. Med Sci (Paris) $2005 ; 21: 947-53$.

\title{
NOUVELLE
}

\section{Elongator orchestre la neurogenèse du cortex cérébral}

Catherine Creppe, Lina Malinouskaya, Marie-Laure Volvert, Pierre Close, Sophie Laguesse, Magali Gillard, Alain Chariot, Laurent Nguyen

\author{
L. Malinouskaya, M.L. Volvert, S. Laguesse, L. Nguyen : \\ Interdisciplinary Cluster for Applied Genoproteomics \\ (GIGA-R), Developmental Neurobiology Unit, \\ University of Liege, CHU Sart Tilman, Liège 4000, Belgique. \\ C. Creppe, P. Close, M. Gillard, A. Chariot : \\ Interdisciplinary Cluster for Applied Genoproteomics \\ (GIGA-R), GIGA Signal Transduction, Liège 4000, Belgique. \\ alain.chariot@ulg.ac.be \\ Inguyen@ulg.ac.be
}

\section{Elongator, un rôle initial dans l'élongation des ARNm}

La dysautonomie familiale, ou syndrome de Riley-Day, est une maladie neurodégénérative héréditaire particulièrement représentée dans la population juive ashkénaze (une personne sur vingt-sept est porteuse de la mutation). Elle est caractérisée par sa transmission autosomique récessive et affecte notamment le développement et la survie de certains neurones des systèmes nerveux autonome et sensoriel. Les patients atteints de cette maladie présentent des troubles gastro-intestinaux, des problèmes de déglutition, des crises de vomissements, de l'hypotonie et de l'instabilité thermique [1]. Ces patients sont également sujets à des crises dysautonomiques durant lesquelles certaines régions de leur cortex cérébral présentent une activité neuronale anormale [2].

La cause moléculaire de cette maladie est une mutation ponctuelle du gène IKBKAP (inhibitor of kappa light polypeptide gene enhancer in B-cells, kinase complex-associated protein) qui code pour la protéine Elpl, laquelle est requise pour l'assemblage du complexe Elongator. Ce dernier est composé de six sous-unités nommées Elpl à Elp6. II a été initialement décrit chez la levure comme interagissant avec la forme hyperphosphorylée de I'ARN polymérase II [3]. Les recherches qui ont suivi ont révélé qu'Elongator contribue à l'élongation de certains ARN messagers. $\varepsilon n$ effet, le complexe Elongator, et plus particulièrement sa sous-unité $\varepsilon \mid p 3$, possède une activité histone acétyltransférase envers l'histone $\mathrm{H} 3$, rendant ainsi possible la progression de I'ARN polymérase II au niveau de la région transcrite de certains gènes $[4,5]$. Des études récentes ont démontré qu'Elongator remplit aussi des fonctions dans le cytoplasme, comme la régulation de l'exocytose et la modification de certains ARN de transfert $[6,7]$.

\section{Elongator,}

la première enzyme responsable

de l'acétylation de la tubuline alpha

Dans ce contexte, les travaux menés dans notre laboratoire ont révélé une nouvelle fonction du complexe Elongator durant la mise en place du cortex cérébral de la souris. En effet, nos résultats montrent qu'Elongator coordonne la migration et la différenciation terminale des neu- rones de projection du cortex cérébral via la promotion de l'acétylation de la tubuline alpha dans le cytoplasme [8]. Plus précisément, afin de caractériser les rôles d'Elongator au cours du développement cortical, nous avons utilisé la technique d'électroporation in utero et démontré que la réduction d'expression de Elpl ou de Elp3 par ARN interférence dans le cortex cérébral d'embryon de souris entraîne un retard de migration des neurones de projection du cortex cérébral. De plus, ces neurones présentent des défauts permanents de formation de neurites caractérisés par une réduction de la longueur totale et une diminution de la complexité de l'arbre dendritique et des axones. L'intégrité du complexe semble par ailleurs requise pour ces fonctions puisque la coexpression d'une forme tronquée de Elpl, qui n'interagit pas avec $\varepsilon l p 3$, ne corrige pas ce phénotype résultant de l'inhibition d'expression endogène d'Elpl par ARN interférence.

Nous avons ensuite caractérisé la voie de signalisation moléculaire qui agit en aval d'Elongator afin de réguler la migration et la différenciation des neurones de projection corticaux. Nos expériences ont 


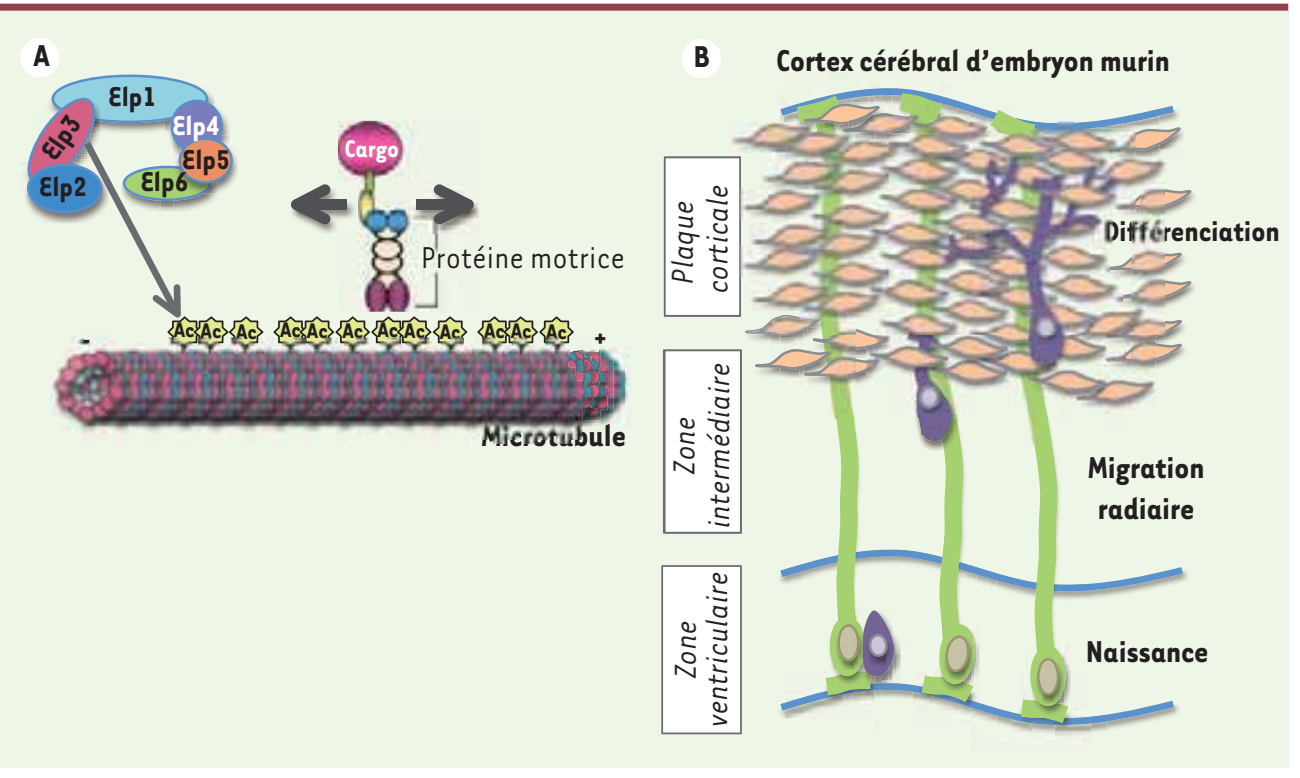

Figure 1. Fonctions de la protéine Elongator. A. Elongator promeut l'acétylation de la tubuline alpha dans les microtubules (Ac). Cette modification post-traductionnelle est importante pour la reconnaissance et l'ancrage de protéines motrices qui transportent divers cargos le long des microtubules. B. Schéma représentant une portion $\mathrm{du}$ cortex cérébral d'un embryon de souris au $14^{\mathrm{e}}$ jour de gestation. Les neurones de projection (mauves) naissent dans la zone ventriculaire à partir de cellules de la glie radiaire (vert) puis migrent le long des fibres radiaires afin d'atteindre la plaque corticale où ils se différencient et développent des dendrites et des axones.

non seulement révélé un enrichissement d'Elpl dans les fractions microtubulaires de neurones corticaux, mais également une interaction entre la tubuline alpha, ou sa forme acétylée, et les sous-unités Elpl et Elp3 d'Elongator. De plus, des cellules déficientes pour Elpl ou Elp3 ont un taux réduit de tubuline alpha acétylée, suggérant que l'activité enzymatique du complexe Elongator est requise pour l'acétylation de la tubuline alpha. Nous avons finalement démontré qu'Elp3 possède une activité enzymatique acétyl-transférase intrinsèque envers la tubuline et, de ce fait, découvert la première enzyme responsable de l'acétylation de cette protéine.

\section{Elongator,}

un partenaire essentiel du transport protéique dans les neurones?

La réduction de l'acétylation des microtubules pourrait expliquer, en partie du moins, les défauts observés dans les neurones déficients pour Elongator. $\varepsilon n$ effet, l'acétylation de la tubuline alpha favorise la liaison et la motilité de moteurs moléculaires comme les kinésines et le complexe dynéine/dynactine sur le réseau microtubulaire de la cel- lule $[9,10]$. Ces protéines transportent des molécules dites «cargo »le long des microtubules afin de les distribuer dans différents compartiments subcellulaires. Ce système de transport s'avère extrêmement important dans les cellules comme les neurones qui, par leur grande taille, posent un véritable défi à l'organisme pour l'organisation du transport intracellulaire [11]. Dès lors, nous suggérons que grâce à sa capacité d'acétyler la tubuline alpha, Elongator régulerait indirectement le transport de protéines requises pour la motilité cellulaire et la différenciation des neurones corticaux.

L'ensemble de nos travaux suggère que certains aspects de la dysautonomie familiale pourraient être expliqués par un défaut de migration des cellules déficientes pour le complexe Elongator [4]. $\varepsilon$ n effet, nous avons initialement montré une altération de la motilité cellulaire en raison d'un défaut d'acétylation des histones $\mathrm{H} 3$ au niveau de la région à transcrire de certains gènes codant pour des protéines impliquées dans la migration. Notre étude suggère maintenant que l'activité acétyl-transférase d'Elongator s'exerce également sur la tubuline alpha. L'acétylation de ce nouveau substrat d'Elongator est une modification post-traductionnelle importante pour la migration des neurones de projection du cortex cérébral. Nos résultats n'excluent cependant pas l'existence de cibles cytoplasmiques supplémentaires d'Elongator, dont l'absence ou la réduction d'acétylation pourrait contribuer à la pathogenèse des patients atteints de dysautonomie familiale. $\diamond$

Elongator orchestrates cerebral cortical neurogenesis

\section{CONFLIT D'INTÉRÊTS}

Les auteurs déclarent n'avoir aucun conflit d'intérêts concernant les données publiées dans cet article.

\section{RÉFÉRENCES}

1. Axelrod FB. Familial dysautonomia. Muscle Nerve $2004 ; 29: 352-63$.

2. Slaugenhaupt SA, Blumenfeld A, Gill SP, et al. Tissuespecific expression of a splicing mutation in the IKBKAP gene causes familial dysautonomia. Am J Hum Genet $2001 ; 68: 598-605$.

3. Otero G, Fellows J, Li Y, et al. Elongator, a multisubunit component of a novel RNA polymerase II holoenzyme for transcriptional elongation. Mol Cell 1999 ; 3: 109-18.

4. Close P, Hawkes N, Cornez I, et al. Transcription impairment and cell migration defects in elongatordepleted cells: implication for familial dysautonomia Mol Cell 2006 ; 22 : 521-31. 
5. Gilbert C, Kristjuhan A, Winkler GS, Svejstrup JQ. Elongator interactions with nascent mRNA revealed by RNA immunoprecipitation. Mol Cell 2004 ; 14 : 457-64.

6. Huang $B$, Johansson MJ, Bystrom AS. An early step in wobble uridine tRNA modification requires the Elongator complex. RNA $2005 ; 11$ : 424-36.

7. Rahl PB, Chen CZ, Collins RN. Elplp, the yeast homolog of the FD disease syndrome protein, negatively regulates exocytosis independently of transcriptional elongation. Mol Cell $2005 ; 17: 841-53$.

8. Creppe C, Malinouskaya L, Volvert ML, et al. Elongator controls the migration and differentiation of cortical neurons through acetylation of alpha-tubulin. Cell 2009; 136: 551-64.

9. Dompierre JP, Godin JD, Charrin BC, et al. Histone deacetylase 6 inhibition compensates for the transport

\section{NOUVELle}

\section{Une cytokine chimérique au secours des maladies auto-immunes}

Moutih Rafei, Jacques Galipeau deficit in Huntington's disease by increasing tubulin acetylation. J Neurosci $2007 ; 27: 3571-83$.

10. Reed NA, Cai D, Blasius TL, et al. Microtubule acetylation promotes kinesin-1 binding and transport. Curr Biol 2006; $16: 2166-72$.

11. Millecamps S, Julien JP. Dysfunction of axonal transport in neuropathies and motor neuron diseases. Med Sci (Paris) $2008 ; 24: 65-71$.
M. Rafei : Institut de recherche en immunothérapie du cancer,

Université de Montréal, CP 6128 ,

Succursale centre-ville, Montréal, Québec, H3C 3J7 Canada.

J. Galipeau :

Division d'hématologie/oncologie, Hôpital général juif, Université McGill, 3755, chemin de la Côte Sainte-Catherine, Montréal, Québec, H3T 1E2 Canada. jacques.galipeau@mcgill.ca.

\section{Bifonctionnalité synergique d'une} cytokine chimérique GM-CSF - IL-2 Les cytokines, notamment le granulocyte colony-stimulating factor (G-CSF) et le granulocyte-macrophage colonystimulating factor (GM-CSF), l'interféron (IFN)- $\alpha$ et I'interleukine-(IL) 2, sont régulièrement utilisées à des fins thérapeutiques en oncologie et, plus particulièrement, dans le traitement du mélanome et de l'hypernéphrome (ou cancer du rein à cellules claires) [1]. Par ailleurs, les interférons et les interleukines sont prisés pour leurs propriétés immunostimulatrices, utiles pour bloquer le développement des tumeurs et pouvant, à l'occasion, entraîner leur régression. Ces données nous ont amenés à proposer le concept selon lequel une cytokine chimérique née de la fusion de deux protéines immunostimulatrices distinctes - une fusokine douée d'une bifonctionnalité synergique serait capable de stimuler simultanément les réponses immunes innées et adaptives. En effet, nous avons déjà démontré que la fusion du GM-CSF à l'IL-2 (GIFT2) confère à cette protéine chimérique des propriétés pro-inflammatoires [2]. Forts de ce succès, nous avons produit une fusokine GIFT de seconde génération mariant le GM-CSF à I'IL-15 (GIFT15). Comme I'IL-15 a été décrite comme stimulant fortement l'immunité innée, nous nous attendions à ce que GIFT15 soit pro-inflammatoire. À notre grande surprise, son action s'est avérée plutôt fortement immunosuppressive [3]. En effet, une analyse de la fonctionnalité de GIFT15 a démontré que cette fusokine se lie au récepteur de l'IL-15 et déclenche une signalisation aberrante en aval caractérisée par une hyperactivation de STAT3 [3]. Toutes les cellules ayant un rôle dans l'immunité, qu'elles soient lymphoïdes ou myéloïdes, qui arborent le récepteur de l'IL-15 perdent leur capacité à participer à une réaction immune normale quand elles sont exposées à GIFT15. Ces effets ont été testés chez des souris possédant un système immunitaire intact et chez lesquelles des cellules tumorales humaines ont été greffées. Contrairement aux souris qui n'ont pas reçu de GIFT15, qui ont rejeté le greffon cellulaire, les animaux traités avec la fusokine ont toléré la greffe tumorale [3]. Ces résultats suggèrent que GIFT15 exerce une fonction suppressive de l'alloréactivité lors de greffes tissulaires non apparentées, voire permet même de tolérer des xénogreffes.

\section{Effet thérapeutique}

de la fusokine GM-CSF - IL-15

dans les maladies auto-immunes

Ce résultat prometteur nous a incités à tester l'effet de GIFTI5 dans le traitement de maladies auto-immunes associées causées par l'activation de cellules $T$ autoréactives. Tel est le cas de la sclérose en plaques [4]. Plusieurs stratégies sont utilisées dans le traitement de cette maladie: l'interféron $\beta$ (IFN- $\beta$ ), l'acétate de glatiramère, le mitoxantrone et le natalizumab $[4,11]$. Toutefois, en dépit des résultats encourageants chez les patients, l'efficacité de ces thérapies est partielle, atténuant la progression de la maladie. Une approche de thérapie cellulaire (testée dans des modèles animaux) consiste à administrer des cellules suppressives telles que les cellules T régulatrices [5], qui peuvent exercer un effet suppressif persistant en provoquant la sécrétion, chez l'hôte, de facteurs inhibiteurs sans induire d'effets secondaires [5]. Les lymphocytes B suppresseurs (désignés aujourd'hui sous le nom de cellules $B$ reg) possèdent aussi une excellente capacité à inhiber l'inflammation due à leur sécrétion d'IL-10, une cytokine anti-inflammatoire [6]. Par exemple, l'induction d'une encéphalite allergique expérimentale $(\varepsilon A \varepsilon)$, un modèle animal

\footnotetext{
${ }^{1}$ Le glatiramère est un copolymère synthétique qui agirait sur les cellules de l'immunité, le mitoxantrone un immunosuppresseur, et le natalizumab ou Tysabri® un anticorps monoclonal humanisé dirigé contre la chaîne $\alpha 4$ des intégrines et bloquant la migration des lymphocytes via la barrière hématoencéphalique.
} 